\title{
The Electron Drift Instrument for MMS
}

\author{
R.B. Torbert ${ }^{1,2}$ - H. Vaith ${ }^{1}$ M. Granoff ${ }^{1}$ - M. Widholm ${ }^{1}$ - J.A. Gaidos ${ }^{1}$ - B.H. Briggs ${ }^{1}$

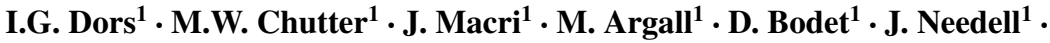 \\ M.B. Steller ${ }^{3}$ - W. Baumjohann ${ }^{3}$ - R. Nakamura ${ }^{3}$. F. Plaschke ${ }^{3}$ - H. Ottacher ${ }^{3}$. \\ J. Hasiba ${ }^{3}$ - K. Hofmann ${ }^{3}$ C.A. Kletzing ${ }^{4}$ - S.R. Bounds ${ }^{4}$ - R.T. Dvorsky ${ }^{4}$ K. Sigsbee $^{4}$ \\ V. Kooi ${ }^{4}$
}

Received: 30 April 2015 / Accepted: 26 June 2015 / Published online: 8 August 2015

(C) The Author(s) 2015. This article is published with open access at Springerlink.com

\begin{abstract}
The Electron Drift Instrument (EDI) on the Magnetospheric Multiscale (MMS) mission measures the in-situ electric and magnetic fields using the drift of a weak beam of test electrons that, when emitted in certain directions, return to the spacecraft after one or more gyrations. This drift is related to the electric field and, to a lesser extent, the gradient in the magnetic field. Although these two quantities can be determined separately by use of different electron energies, for MMS regions of interest the magnetic field gradient contribution is negligible. As a by-product of the drift determination, the magnetic field strength and constraints on its direction are also determined. The present paper describes the scientific objectives, the experimental method, and the technical realization of the various elements of the instrument on MMS.
\end{abstract}

Keywords Magnetospheric Multiscale $\cdot$ Reconnection · Magnetospheric physics

\section{Introduction}

The precise measurement of electric and magnetic fields in the rarefied plasmas within reconnection regions is the primary objective of the FIELDS suite on MMS. The EDI instrument, one of the six sensors within the suite (Torbert et al. 2014, this issue) measures these fields by tracking the drift of a test electron beam which gyrates far from the spacecraft in the ambient magnetic and electric fields, and thus is immune to many local spacecraft disturbances. By combining the accuracy of the EDI technique with the time resolution of the double-probe technique, as used by the Spin-Plane Double Probes (SDP) and Axial Double

\footnotetext{
R.B. Torbert

roy.torbert@unh.edu

1 University of New Hampshire, Durham, NH 03824, USA

2 Southwest Research Institute, San Antonio, TX 78227, USA

3 Space Research Institute, 8042 Graz, Austria

4 University of Iowa, Iowa City, IA 52242, USA
} 
Probe (ADP) sensors on MMS, a high fidelity determination of the 3D electric field is possible for the first time in space plasma studies. Our understanding of reconnection can only be advanced with a reliable measurement of this $3 \mathrm{D}$ field. In particular, the presence of a significant component of electric field parallel to the magnetic field may be a distinguishing feature of reconnection.

The operational principle of the EDI technique was originally proposed by Melzner et al. (1978) and proven on ESA's GEOS spacecraft. This instrument was limited to spin period time resolution and could be operated only for small angles between the magnetic field vector and the spacecraft spin axis. The next generation of EDI instruments on Equator-S and Cluster (see Paschmann et al. 1998) could determine the electric field for any direction of the ambient magnetic field and was shown to be highly accurate even in the presence of photoelectron and wake asymmetries that often occur around spacecraft in rarefied plasmas. The EDI instruments on MMS represent the third generation of this technique with higher tracking bandwidth and more thoroughly characterized detectors than those flown on Cluster.

\section{Scientific Objectives}

The ability of the EDI instrument to make accurate and highly sensitive measurements of the electric field and of the perpendicular gradient of the magnetic field makes possible a variety of studies that comprise the essence of the MMS mission.

MMS is designed primarily to study small-scale structures in three dimensions around reconnection sites in the Earth's plasma environment, specifically in the dayside magnetopause regions and in the magnetotail. The reconnection processes leading to the formation of such structures are fundamental to the key processes of interaction between the solar wind and the magnetospheric plasmas. Within reconnection regions, plasmas are accelerated and heated by mechanisms that are still not well understood but must surely involve electric fields. In particular, acceleration by parallel electric fields may be an indicator of reconnection (Egedal et al. 2012; Pritchett and Mozer 2009). We refer the reader to the companion papers in this issue (Burch et al. 2014; Torbert et al. 2014) for an account of the full MMS objectives and the important roles of magnetic and electric fields in understanding reconnection. The accuracy by which MMS can measure these fields dictates whether specific plasma and field properties can be identified unambiguously. Examples include the presence of parallel fields, plasmas flowing with the $\mathbf{E} \times \mathbf{B}$ velocity ("frozen-in" flows), and magnetic reconnection. EDI is able to measure these fields with minimal disturbance from local spacecraft effects because the electron beam gyrates far from its origin. The fields are then calculated by a purely geometric algorithm from the drift of the beam in one or more gyro periods, as described below. On MMS, the measurements of magnetic fields from the magnetometers (flux-gates and search coil) and electric fields from the double-probe sensors are calibrated and adjusted with the use of these values from EDI. In addition, EDI provides very high time resolution electron flux measurements (at millisecond cadence) at a single, but programmable energy. This energy is the energy of the test electron beam, when EDI is operated in "electric field" mode, but can be varied if EDI is placed in the "ambient" mode. These high time resolution measurements can be extremely useful for the identification of fast moving plasma boundaries, when used to complement those of the normal electron spectrometers on MMS.

For the EDI instrument, Table 1 lists the quantities that can be obtained from the measured electric fields and magnetic field gradients, and the information that can be derived. 
Table 1 Derivable information

\begin{tabular}{ll}
\hline Tools & Information \\
\hline $\mathbf{E}^{i}+\left(\mathbf{v}^{i} \times \mathbf{B}^{i}\right)$ & Resistivity; deviation from frozen flux condition \\
$\mathbf{E}^{i} \cdot \mathbf{I}$ & Conversion of electromagnetic energy \\
Variance analysis of $\mathbf{E}^{i}(t)$ & Attitude and motion of boundaries; normal and tangential fields \\
Least squares fit of $\mathbf{E}^{i}(t)$ & De Hoffmann-Teller frame; intrinsic electric fields \\
$\int_{t_{1}}^{t_{2}} \mathbf{E}^{i}(t) \cdot \mathbf{u} d t$ & Potential difference across layer of discontinuity \\
$\int_{t_{1}}^{t_{2}} \nabla_{\perp} B^{i}(t) \cdot \mathbf{u} d t-\left[B^{i}\left(t_{2}\right)-B^{i}\left(t_{1}\right)\right]$ & Measure of stationarity of magnetic field profiles \\
$\nabla_{\perp} B^{i}(t) \cdot \mathbf{s}^{i j}-\left[B^{j}(t)-B^{i}(t)\right]$ & Comparison of small and large scale magnetic gradients \\
$\left\langle E_{x}^{i}(t) E_{y}^{j}(t+\tau)\right\rangle$ & From this and other correlations: characterization of turbulence \\
$\nabla \cdot \mathbf{E}$ & Shear flows \\
$\nabla \times \mathbf{E}$ & $\partial \mathbf{B} / \partial t$; Induction electric fields \\
\hline
\end{tabular}

Superscripts $i, j$ indicate the four spacecraft

$\mathbf{u}$ denotes boundary velocity, $\mathbf{s}^{i j}$ denotes inter-spacecraft separation

Vector derivatives are approximated by finite differences between quantities measured at the different spacecraft locations

One of the important objectives of the MMS mission is to obtain differential quantities by measurements of particle and field properties at the four spacecraft locations surrounding reconnection regions. These differences can be used to form quantities such as the gradient, curl, and divergence of the fields, and of the plasma moments such as velocity and pressure. These differentials will yield other physical properties such as current densities, vorticity flow, shear flows, induction electric fields, and momentum balance from the divergence of the pressure and magnetic stress tensors.

EDI can be used to obtain an estimate of the magnetic field gradient in the plane perpendicular to the magnetic field. This could be a useful supplement to the differentials obtained from measurements made at the four spacecraft. The spacecraft separation will in general be much larger (tens to a few hundreds of $\mathrm{km}$ ) than the electron gyro radius (less than $1-3 \mathrm{~km}$ ), which is the scale on which EDI will measure B. Comparing the two differentials will allow a test of the consistency of the differentials over the two scales. This feature was implemented on Cluster, and could be on MMS, but at present, it is not anticipated to be required because of the much smaller spacecraft separation on MMS.

In the next section, we describe how EDI measures the electric and magnetic fields. As the MMS manifestation of EDI is very similar to that of Cluster, much of the following discussion can be found in Paschmann et al. (1998). The description below is more detailed where MMS differs from Cluster.

\section{Principle of Operation}

In the presence of a homogeneous magnetic field $\mathbf{B}$ and a force $\mathbf{F}_{\perp}$ perpendicular to the magnetic field, charged particles perform a circular motion with gyro period $T_{g}$, superimposed with a constant drift velocity $\mathbf{V}_{d}$ that is given by

$$
\mathbf{V}_{d}=\frac{1}{q} \frac{\mathbf{F}_{\perp} \times \mathbf{B}}{B^{2}}
$$


Fig. 1 Drift parameters for $1 \mathrm{keV}$ electrons

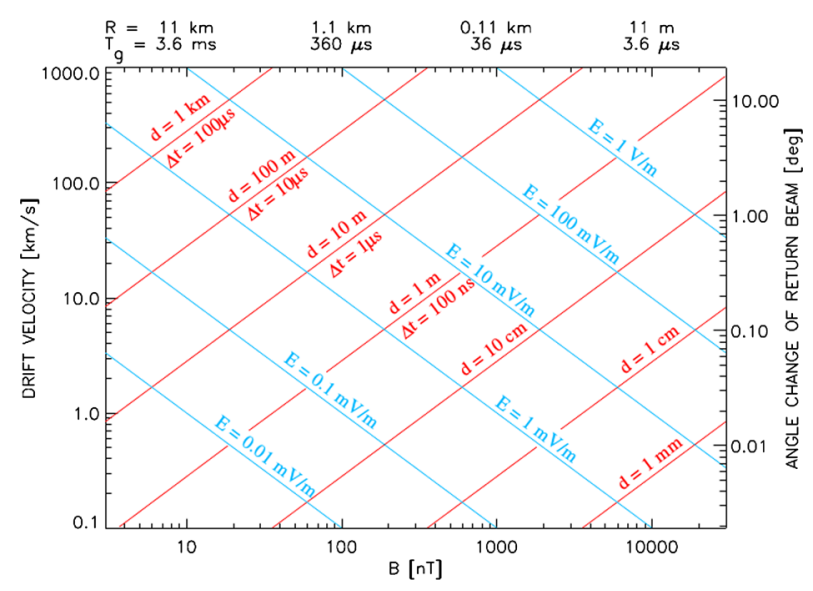

If $\mathbf{F}_{\perp}$ is caused by an electric field $\mathbf{E}_{\perp}$ then

$$
\mathbf{V}_{d}=\frac{\mathbf{E}_{\perp} \times \mathbf{B}}{B^{2}}
$$

Due to the drift, the particles are, after one gyration, displaced from their origin by the drift step $\mathbf{d}$ which is related to the drift velocity by

$$
\mathbf{d}=\mathbf{V}_{d} T_{g}
$$

A measurement of the drift step, together with the gyro period, thus allows the determination of the drift velocity.

The Electron-Drift Instrument employs two complementary techniques to measure this drift step. The basis is the injection of two weak electron beams perpendicular to the ambient magnetic field $\mathbf{B}$ and their detection after they have performed one or more gyrations. The first method uses the fact that, due to the drift superimposed on the circular motion, the electrons no longer return to their origin, unless they are fired in unique directions. Two such directions exist at any given moment if the drift velocity is smaller than the initial velocity of the electrons at the time of their injection. Finding these directions, the drift step $\mathbf{d}$ can be determined using a triangulation technique. The second method is based on the different lengths of the two paths along which the electrons travel when emitted in the two directions, and on the difference in the associated times of flight. This difference is proportional to the magnitude of the drift step. The magnitudes of the relevant parameters for both triangulation and times of flight are diagrammed in Fig. 1, where the magnitude of $\mathbf{B}$ is the abscissa and the drift velocity of electrons is the ordinate.

\subsection{Triangulation Technique}

As seen in Fig. 2, in the presence of a drift due to a perpendicular force $\mathbf{F}_{\perp}$ electrons emitted from a common source point $S$ into any direction perpendicular to $\mathbf{B}$ will, after one gyration, be focused at a point $D$ that is displaced from $S$ by the drift step d. A detector located at $D$ would detect these electrons. Since $\mathbf{d}$ is the quantity to be measured, it is not possible to position an electron source at $S$. This is not necessary, however. In order to hit a detector at $D$, it is sufficient to let an arbitrarily located electron gun aim a narrow beam such that the beam appears to emanate from $S$. Using two guns at different positions, the source point $S$ can be found from triangulation of the emission directions. 
Fig. 2 Principle of drift triangulation

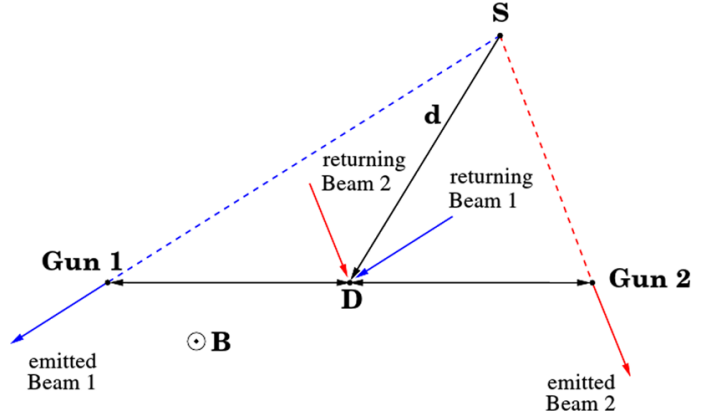

Fig. 3 Triangulation scheme for the two gun/detector units

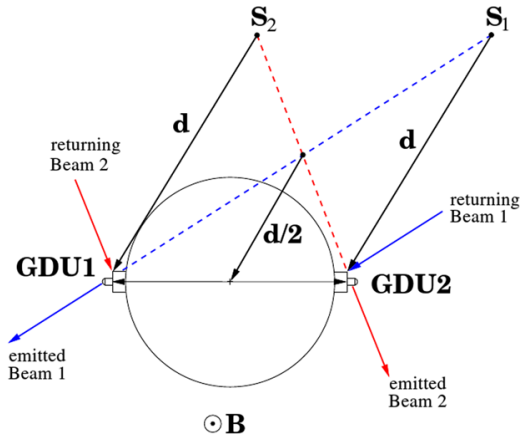

$\odot \mathbf{B}$

The actual configuration on both Cluster and MMS is diagrammed in Fig. 3, where there are two "Gun-Detector-Units", GDU's, each containing a gun source and a corresponding detector. Although this means that the effective baseline is twice the separation, the principle is the same. Unlike on Cluster, the GDUs are not oriented to have their symmetry axes pointing radially outward from the SC center, but there is a 4 degree tilt in order to obtain a better field-of-view. A consequence of this slight tilt angle is that there is no common symmetry axis of the two GDUs. The symmetry axes are still parallel, however. While this has no impact on the operation of the instrument, it must be taken into account in ground processing.

\subsection{Drift Velocities from Time-of-Flight Measurements}

The virtual source point is also referred to as the "target". Having the guns located at any other point than $\mathrm{S}$ has several consequences. First, neither $S$ nor $D$ are any longer focal points of the beams. Second, the travel times between gun and detector no longer equal the gyro time, $T_{g}$, but are shorter (longer) than $T_{g}$ if the beam is directed "away" from ("towards") $S$. The situation is best illustrated by considering the trajectories in a frame of reference in which there is no electric field and thus no drift, as illustrated in Fig. 4. In this frame, the spacecraft is moving with the drift velocity $\mathbf{V}_{d}$, and the beam trajectories are circles. At any given point in time, two firing directions $\mathbf{e}_{1}$ and $\mathbf{e}_{2}$ exist that will cause beams emitted at time $t_{0}$ along these directions to hit the spacecraft at times $t_{1}$ and $t_{2}$ after slightly less $\left(\mathbf{e}_{1}\right)$ and more $\left(\mathbf{e}_{2}\right)$ than one gyration, respectively. The difference $t_{2}-t_{1}$ between the times of flight of the "away" and "towards" beams yields the magnitude of the drift step. A consequence that is not apparent from the straight line approximation used in Fig. 3 is that 
Fig. 4 Principle of drift determination via time-of-flight measurements

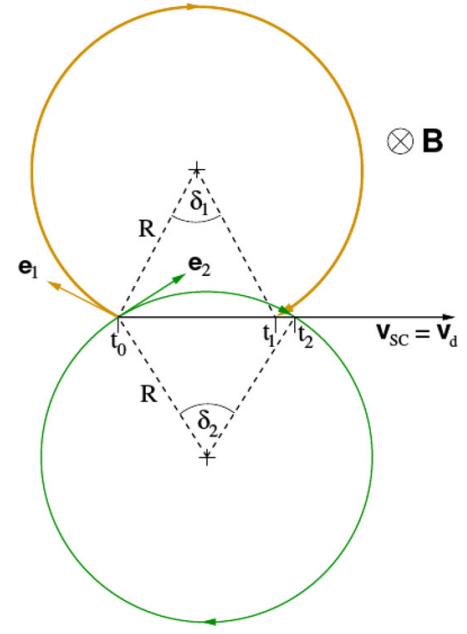

Table 2 Characteristic quantities for key regions

\begin{tabular}{|c|c|c|c|c|c|c|}
\hline Parameter & $\begin{array}{l}\text { Solar } \\
\text { wind }\end{array}$ & $\begin{array}{l}\text { Magneto- } \\
\text { sheath }\end{array}$ & $\begin{array}{l}\text { Magneto- } \\
\text { pause }\end{array}$ & $\begin{array}{l}\text { Tail } \\
\text { lobe }\end{array}$ & $\begin{array}{l}\text { Plasma- } \\
\text { sheet }\end{array}$ & $\begin{array}{l}\text { Ring } \\
\text { current }\end{array}$ \\
\hline Magnetic field, $B$, nT & 8 & 40 & 40 & 30 & 20 & 300 \\
\hline Electric field, $E, \mathrm{mV} \mathrm{m}^{-1}$ & 3.6 & 8.0 & 4.0 & 0.5 & 1.0 & 1.0 \\
\hline $\begin{array}{l}\text { Electron gyroradius, } \\
R_{g}, \mathrm{~km}\end{array}$ & 13 & 3 & 3 & 4 & 5 & 0.4 \\
\hline $\begin{array}{l}\text { Electron gyrotime, } \\
T_{g}, \mathrm{~ms}\end{array}$ & 4.5 & 0.9 & 0.9 & 1.2 & 1.8 & 0.1 \\
\hline Drift step, $d, \mathrm{~m}$ & 2000 & 179 & 89 & 20 & 89 & 0.4 \\
\hline ToF difference, $\Delta T, \mu$ s & 214 & 19 & 9 & 2 & 9 & 0.04 \\
\hline Angle change, $\delta$, deg & 8.7 & 3.9 & 1.9 & 0.3 & 1.0 & 0.1 \\
\hline Ambient diff. E-flux & $1.0 \times 10^{5}$ & $1.0 \times 10^{6}$ & $1.0 \times 10^{7}$ & $1.0 \times 10^{5}$ & $2.0 \times 10^{7}$ & $1.0 \times 10^{8}$ \\
\hline Beam current, nA & 1000 & 200 & 300 & 30 & 400 & 0.5 \\
\hline Optics state & 7 & 7 & 7 & 7 & 7 & 4 \\
\hline Ambient count rate, $\mathrm{s}^{-1}$ & $2.2 \times 10^{3}$ & $2.2 \times 10^{4}$ & $2.2 \times 10^{5}$ & $2.2 \times 10^{3}$ & $4.4 \times 10^{5}$ & $1.4 \times 10^{5}$ \\
\hline Beam count rate, $s^{-1}$ & $1.6 \times 10^{4}$ & $1.8 \times 10^{5}$ & $5.4 \times 10^{5}$ & $1.8 \times 10^{5}$ & $3.6 \times 10^{5}$ & $5.6 \times 10^{5}$ \\
\hline Contrast & 7.2 & 8.1 & 2.5 & 82 & 0.8 & 4.0 \\
\hline Signal-to-noise ratio & 10.6 & 38.0 & 36.5 & 122 & 17.2 & 47.2 \\
\hline
\end{tabular}

Table values are for $1 \mathrm{keV}$ electrons

Contrast and signal-to-noise ratios are for $1 \mathrm{~ms}$ samples. Beam and background count rates are computed for the collection areas $A$ and geometric factors $H$ associated with the chosen Optics State (see Table 3 )

the directions of a beam emitted by a gun and received by the detector after one gyration are not parallel, but subtend a small angle, $\delta_{1}$ and $\delta_{2}$ for firing directions $\mathbf{e}_{1}$ and $\mathbf{e}_{2}$, respectively. These "aberration" angles are greatly exaggerated in Fig. 4 for purposes of illustration of the principle. Typical values are given in Table 2. 


\subsection{Measurement of $B$}

The gyro period can be obtained from the mean of the two travel times, one "toward" and one "away" from the target, as described above:

$$
T_{g}=\frac{t_{1}+t_{2}}{2}
$$

From $T_{g}$ the magnetic field strength, $B$, is obtained via

$$
T_{g}=\frac{2 \pi m \gamma}{e} B
$$

Values of $T_{g}$ range from about 0.1 to $5 \mathrm{~ms}$ (see Table 2). The method is sensitive enough to require the relativistic $\gamma$ correction even for a $500 \mathrm{eV}$ electron. When times of flight are measured, the magnetic field strength is determined with very high accuracy, e.g., to within $0.1 \%$ for a $30 \mathrm{nT}$ field. This feature is used for an accurate in-flight determination of the flux-gate magnetometer offsets.

\subsection{Return Beam Intensities}

The flux of returning beam electrons incident on the detector depends upon many factors, including the angular current distribution of the outgoing beam, the beam gyro radius, possible beam modification by electrostatic or wave-particle forces, and the geometrical arrangement of the gun and detector with respect to the drift step vector. The outgoing beam has an opening angle, $\alpha$, of approximately $1^{\circ}$. Thus the beam diverges along the magnetic field direction by a distance $s_{\|}=2 \pi R_{g} \alpha / 57.3$, where $R_{g}$ is the gyro radius, but is focused in the plane perpendicular to $\mathbf{B}$ after one gyro orbit. By definition, this focus is located one 'drift step' from the gun. In general, beam electrons with the proper firing direction encounter the detector either somewhat before, or somewhat after, this focus point. Because of the angular divergence both along and perpendicular to $\mathbf{B}$, the detector intercepts only a very small part of the emitted beam.

Equation (5) (Quinn et al. 1979) gives the beam flux, $F$, in $\mathrm{cm}^{-2} \mathrm{~s}^{-1}$ at the detector for an emitted beam with a flat current distribution over a square angular cross-section, where $I$ is the gun current in nA, $B$ the magnetic field strength in $\mathrm{nT}, W$ the beam energy in $\mathrm{keV}$, $x$ the distance from the gun's gyro focus to the detector in meters, $\alpha$ the beam full width parallel and perpendicular to $\mathbf{B}$ in degrees, and $R_{g}$ the gyro radius in meters.

$$
F=3.1 \cdot 10^{3} \frac{I B}{x \alpha^{2} W^{1 / 2}\left(1 \pm x / 2 \pi R_{g}\right)}
$$

The \pm -term in the denominator accounts for divergence parallel to $\mathbf{B}$ between the gun's gyrofocus and the detector, depending upon whether the detector intercepts the beam before $(-)$ or after $(+)$ the gyrofocus. Of course a square, uniform cross-section is not a realistic representation of the actual beam. However for that portion of the real beam that has the same angular current density $I / \alpha^{2}$ as the uniform beam, the return flux at the detector would be the same.

The beam divergence leads to a large variation in the return beam flux with magnetic field intensity and drift step. In order to compensate partially for this variation, both the beam current and the detector optics are adjusted by EDI's controller unit. The optics may be commanded into a number of different 'states' (see Sect. 4.2). These states allow a good deal of flexibility in the choice of the detector's effective area $(A)$ to the return beam and its geometric factor $(H=G \Delta E / E)$ to ambient electrons. 
Table 2 illustrates sample values of return-beam count rates and signal-to-noise ratios for several regions of interest, using appropriately chosen optics states, which have different effective areas. The values in Table 2 are for a $1 \mathrm{keV}$ beam with a $1^{\circ}$ width. The beam current has been limited to keep the instantaneous count rates (per anode) below approximately $4 \times 10^{5}$ counts/s. The signal-to-noise ratios are based upon counts when the beam is gated on and accumulated over a period of $1 \mathrm{~ms}$. Because $x$, the distance between the beam focus and the detector, depends upon the relative geometry of the guns, detectors, and drift step, we have taken $x$ to be equal to the larger of either the drift step or 2 meters. We have also ignored the beam spreading parallel to the magnetic field that occurs between the focus and the detector, since the sign of this extra term depends upon the specific geometry.

\subsection{Requirements for Gun/Detector Configuration}

In order to accommodate the time-of-flight measurements, there must be two guns, each steerable over a solid angle of $2 \pi$ steradians ( $\mathrm{sr}$ ), but facing opposite hemispheres. As the detectors require active steering into the corresponding directions, two such detectors are needed, each able to cover also $2 \pi$ sr. The time-of-flight technique puts no restriction on the relative location of guns and detectors other than those imposed by field-of-view considerations.

The triangulation technique, on the other hand, requires that guns and detectors are well separated in order to provide adequate triangulation baselines. Ideally, they should not be coplanar, but rather form a tetrahedron. Otherwise there will be situations where the baseline vanishes, i.e., when $\mathbf{B}$ and $\mathbf{v}_{D}$ are in the gun/detector plane. As technical constraints rule out such a tetrahedron solution on Cluster and MMS, one gun and one detector are combined into a single package, and two such packages are mounted on opposite sides of the spacecraft (see Fig. 3). So they are not only coplanar, but even co-linear. As a consequence, the triangulation baseline will vanish each time the projection of the two packages in the plane perpendicular to $\mathbf{B}$ is aligned with $\mathbf{v}_{D}$. Even though this will cause a spin modulation of the accuracy with which the drift step is triangulated, the electron guns will stay on track. Furthermore, the time-of-flight technique, which will always be executed simultaneously with the triangulations, will not be affected at all. (Note that in the worst case of a spin axis perpendicular to both $\mathbf{B}$ and $\mathbf{v}_{D}$, the baseline is always zero.)

\subsection{Beam Recognition, Tracking, and Coding}

The electron drift technique described in the previous sections requires first a scheme capable of initially finding the beam for arbitrary directions of magnetic and electric fields; secondly, a scheme to keep the beam on target, and, finally, a scheme which determines the time-of-flight of the electrons for each beam.

From the various schemes implemented on Cluster only the most advanced one has been implemented on MMS. It is described in more detail in Sect. 6.1. Using onboard real-time flux-gate magnetometer data the beams are swept in the plane perpendicular to $\mathbf{B}$ at constant angular velocity until the return beams are detected. Upon successful detection of the return beams, the emitted beams are swept rapidly back and forth across the target. To measure the time-of-flight and detect the return beams in the presence of background electrons, the beams are modulated with a coded waveform. The time delay between emission and reception is measured by correlating the received signal with a properly delayed copy of the original waveform. 


\subsection{Capabilities and Limitations of the Technique}

The electron drift technique is capable of providing several unique measurements. First, it provides the electric field $\mathbf{E}_{\perp}$ perpendicular to the magnetic field, including its component along the spacecraft spin axis. By contrast, the spin-plane double-probes (SDP), which are the most accurate on MMS, measure $\mathbf{E}_{\perp}$ in the plane of the wire booms only. The ADP sensors provide the third component, but as this is not a spinning component, the accuracy is somewhat less than SDP and requires calibration by EDI. Second, the electron drift technique can provide the unique capability of measuring local magnetic field gradients, $\nabla_{\perp} B$, if necessary. For the magnetic field environments on MMS, this correction is very small and will be seldom used. Third, through its time-of-flight measurements the technique also yields accurate measurements of the magnetic field strength, $B$. Finally, the measurements are essentially unaffected by the presence of the spacecraft. A time resolution of between 10 and 100 measurements per second is possible depending on the detector signal-to-noise ratio.

On the other hand, the electron drift technique is adversely affected by intrinsic beam instabilities, strong scattering of the beam by ambient fluctuations, large-amplitude 'spikes' in the electric field, and very rapid magnetic field variations. Furthermore, there can be signal-to-noise problems as a result of insufficient beam current and/or excessive fluxes of ambient electrons. All these effects can cause a loss of beam track and thus a momentary loss of data.

By combining the time resolution of the double probes and the accuracy of EDI, the FIELDS suite on MMS has the potential to provide revolutionary measurements of electric fields in the plasma regions relevant to reconnection.

\subsection{Spacecraft Potential}

The spacecraft is normally at a potential, $\Phi$, that differs slightly from the ambient plasma potential. As the test electrons traverse the sheath surrounding the spacecraft, they are deflected and consequently enter the region of undisturbed ambient electric field with perturbed initial conditions. Normally, this will lead to an additional displacement of the returning beam. Since the proposed measurement of $\mathbf{E}_{\perp}$ is based on measurements of the direction of the outgoing beam, we best express the perturbation caused by spacecraft fields in terms of the angular deflection of the outgoing beam, $\beta$. Upon return the beam may suffer a similar deflection. It is easy to estimate an upper limit of $\beta$, not taking into account the peculiarities of the field geometry:

$$
\beta<\frac{e \Phi}{4 W}
$$

In sunlight $\Phi$ is of the order of a few tens of volts. With $W=1 \mathrm{keV}$ for the electron beam, the error introduced by this effect is comparable to the pointing accuracy of the beam.

The ASPOC instrument on MMS (see Torkar et al. 2014, this issue) is designed to keep the spacecraft potential at low values in the outer regions of the magnetosphere, magnetosheath, and in the solar wind where normal spacecraft potentials may be several volts positive. This system is based on the emission of indium ions at several $\mathrm{keV}$ energy and with a current of $1-10 \mu \mathrm{A}$, the spacecraft potential will be kept at a low positive potential relative to the ambient plasma. When the potential control system is operating, EDI can use currents up to $10^{-7} \mathrm{~A}$ (or possibly more) without influencing the spacecraft potential in any significant way. The experience on the Cluster mission has shown that this is the case. 
There could be a significant effect of the EDI electron beam current on the spacecraft potential when ASPOC is not operating. The electron beam can be considered to be another 'photoemission' component at an energy of $1 \mathrm{keV}$. It can be seen from the three curves in Fig. 1 of Riedler et al. (1997), for the Cluster spacecraft which is very like MMS in this regard, that it only takes from 10 to $30 \mathrm{~V}$ of spacecraft potential to reduce their assumed photoemission current to about $1 \mu \mathrm{A}$. At higher (positive) spacecraft potentials, an EDI beam current of $1 \mu \mathrm{A}$ could dominate over the photoemission current and drive the spacecraft potential even more positive. As was the experience on Cluster, it is expected in such situations that a much smaller EDI current will be used.

\section{Technical Description}

The essential elements of the instrument are two electron guns, two detectors with their associated analog electronics, high-voltage supplies, digital controls, and correlators; and a controller unit which includes the interfaces with the spacecraft and with other instruments. As illustrated in Fig. 3, guns and detectors are combined in pairs into a single unit, referred to as the gun/detector unit (GDU). The two GDUs are mounted on opposite sides of the spacecraft. For a detailed cutaway view of the GDUs, see Fig. 5.

\subsection{Electron Guns}

In order to be able to aim the beam at the target for arbitrary magnetic and electric field directions, the electron guns must be capable of providing a beam that can be steered rapidly into any direction over more than a hemisphere. Electron energies must be small enough that $\nabla_{\perp} B$ drifts are not significant, but variable to determine this component if necessary. At the same time the energy dispersion must be small to restrict beam spreading in space and time. Beam currents must be kept sufficiently low to avoid instabilities and/or interference with other experiments on the spacecraft. To maximize the return signal in the detectors, the angular width of the beam must be kept small, but still large enough to account for uncertainties in pointing direction. Electron time-of-flight measurements require that the beam be modulated with frequencies up to $4 \mathrm{MHz}$.

A design meeting these requirements was successfully implemented on Cluster, as described in Paschmann et al. (1998). The MMS design is nearly identical. A conventional electron source, consisting of a tungsten cathode and several control electrodes (Wehnelt, Focus, and Anode) is used to produce a narrow beam at 2.7 times the required energy. The electron energy is set by the cathode potential and can be varied between 0.25 and $1.0 \mathrm{keV}$. The spread in energy is determined by the thermal spread $(\approx 0.2 \mathrm{eV})$ and the variation of the potential over the emitting part of the cathode which is $\approx 0.5 \mathrm{eV}$. The beam current is controlled via the current applied to the tungsten filament and can be varied between 0.1 and $2000 \mathrm{nA}$. The beam is intensity-modulated by superposing the code-signal from the correlator onto the static Wehnelt voltage.

After exiting from the anode, the beam is deflected into the desired direction by an octopole arrangement of electrostatic deflectors. The electrons then approach a hightransmission grid at ground potential. This retarding potential decelerates the electrons to their final energy. A maximum deflection angle of $96^{\circ}$ can be achieved, but only up to $93^{\circ}$ will be used due to field-of-view constraints. 
Fig. 5 Cutaway view of the GDU showing key optics surfaces

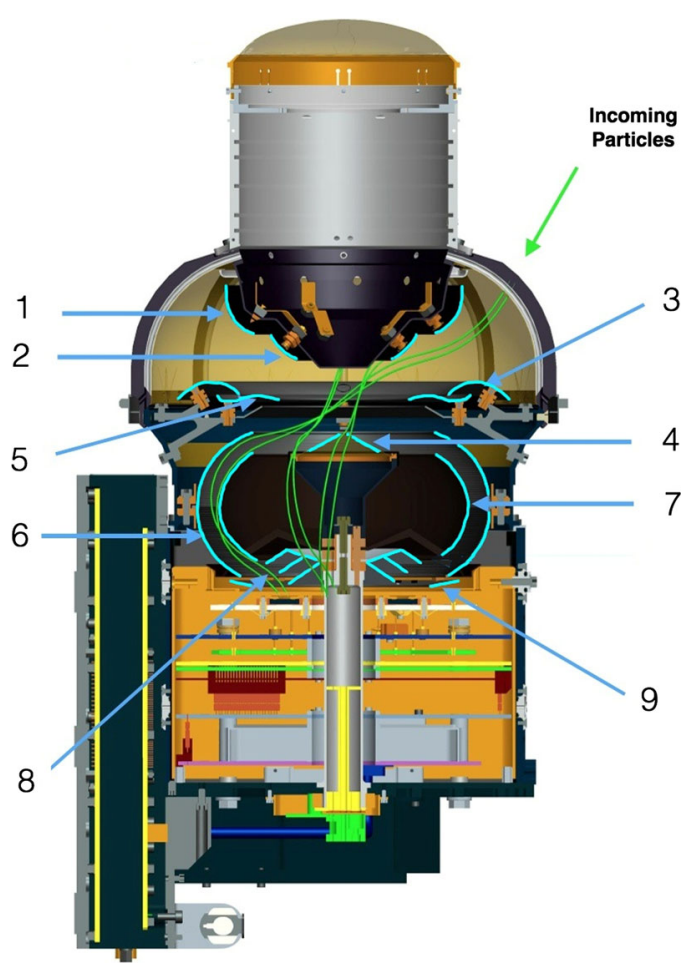

\subsection{Detectors}

The demands of the Electron Drift Instrument require a detector design that is different from normal particle detectors. Not only must it be able to look in any direction within a region greater than a $2 \pi$ sr hemisphere, the detectors must have a large effective area $\left(\sim 2-3 \mathrm{~cm}^{2}\right)$ to compensate for the low returning beam fluxes. Unlike the natural plasma, the returning beam is mono-energetic and unidirectional. Therefore, the signal-to-noise ratio may be improved by designing the detector to be selective in velocity space. To make continuous electric field measurements while either magnetic or electric fields vary rapidly, the detectors must be capable of changing their look directions in less than a millisecond. As they cannot be shrouded in any direction, they must have good internal light rejection.

The detector system that was designed to meet these requirements is illustrated in Fig. 5. It consists of an optics section, a programmable sensor, associated electronics, and voltage generators. Adjustment of the look direction is achieved in elevation by deflecting the incoming beam, and in azimuth by selecting a contiguous set of sectors of the annular imageregion on the sensor micro-channel plate (MCP). The incoming beam of electrons is monoenergetic and mono-directional, and illuminates the entire detector. The large effective area is achieved with double focusing, as explained in Sect. 4.2.1. Here, 'double focusing' refers to the simultaneous concentration of the beam in two angular planes, rather than in the more commonly understood sense of energy and angle.

To cover all beam directions, two identical detectors are mounted such that they view opposite hemispheres. 


\subsubsection{Optics}

To detect the returning electron beam from any direction over $2 \pi$ steradians, the electron optics consist of several shaped potential surfaces, which are controlled by nine separate variable HV supplies. Figure 5 shows a cutaway view which shows the optics and sensor portions of the GDU. The key optics surfaces have been highlighted in light blue to more clearly show their shape and positions. These are numbered as follows: (1) the upper deflector, (2) the upper injector, (3) the lower deflector, (4) the retainer cone, (5) the lower injector, (6) the outer analyzer, (7) the inner analyzer, (8) the suppressor, and (9) the extractor.

The path of electrons traveling through the EDI optics is shown in green traces in Fig. 5.

After passing through the optics aperture screen, the beam electrons encounter a large electric field generated by the two deflector electrodes on each detector. These provide the initial bending of the beam into the nominal entrance direction. The injectors also contribute an electric field with components transverse to the particle trajectory and in the radial direction of the detector's cylindrical coordinates. The injectors provide further refinement in the beam position and direction. As these electrodes are exposed to sunlight, they cannot be biased negatively or they would expel photo electrons that would interfere with other spacecraft experiments. The retainer cone provides additional control of transverse and radial electric fields in the central region of the optics. Because the retainer cone at times may be biased negatively, it is constructed from a wire mesh to minimize the surface area from which photo electrons can emanate.

As illustrated in Fig. 5, the electron beam is focused in cross sections parallel to the symmetry axis ('polar' focusing) near the entrance of the electrostatic analyzer, and again near the exit of the analyzer. In the projection perpendicular to the symmetry axis ('azimuthal' focusing), the beam is partially focused and then diverges before it enters the analyzer, in such a way as to exploit more effectively the final azimuthal focusing that occurs within the analyzer. Together, the deflectors, injectors and retainer cone constitute an 'immersion lens' that projects the beam past the central region into the entrance of the electrostatic analyzer defined by the inner and outer analyzer surfaces, thereby increasing the effective area of the aperture. The azimuthal focusing also extends the width of the effective aperture area and thus reduces the effects of shadowing caused by the gun-support struts.

The deflector potentials play the most important role in determining the polar look direction; the injector potentials are varied primarily to maintain azimuthal beam spread at large deflection angles, but they also influence the look direction. The retainer-cone potential provides additional control over the azimuthal focus and polar look direction.

The inner- and outer-analyzer electrodes select electrons in the desired energy range, and their toroidal shapes also contribute to azimuthal focus, as seen in Fig. 5. Emerging from the electrostatic analyzer, the electrons pass between two additional electrodes: the extractor and the suppressor. These electrodes adjust the radial position of the MCP image and direct the electrons to strike the microchannel plate (MCP) with impact angles closer to the surface normal.

As described above, there are nine potential surfaces requiring nine independently programmable high-voltage supplies to operate the optics subsystem. Although all nine electrode voltages can depend on polar angle, in order to conserve controller resources, only the five that depend most strongly on polar angle (deflectors, injectors and retainer cone) are controlled dynamically.

For a given beam energy and polar angle, different combinations of these voltages can be chosen to obtain different collection areas, $A$, for the beam and different geometrical factors, $H$, for the ambient ('background') electrons (see Table 2). Therefore, with appropriate combinations of voltages we can optimize beam-signal levels or signal-to-noise (SNR) levels, 
Table 3 Optics states

$A$ is the collection area $\left(\mathrm{cm}^{2}\right)$ for beams

$H=G(\Delta E / E)$ is the geometric factor $\left(\mathrm{cm}^{2} \mathrm{sreV} / \mathrm{eV}\right)$ for ambient electrons

Angle is the FWHM acceptance angle in degrees

\begin{tabular}{lllc}
\hline State & $A$ & $H$ & Angle \\
\hline 2 & 2.2 & 0.110 & 20 \\
3 & 2.7 & 0.016 & 7 \\
4 & 0.5 & 0.001 & 4 \\
6 & 5.8 & 0.210 & 10 \\
7 & 1.3 & 0.022 & 14 \\
\hline
\end{tabular}

depending on the circumstances. Also, combinations of these nine voltages can be chosen to achieve other special optical characteristics, such as wide or narrow values of polar-angle acceptance or energy bandpass. For example, the detector's energy bandpass can be adjusted for a given incident particle energy by setting independently the voltage difference between the plates (to control the actual bandpass width) and the average voltage on the analyzer plates (to shift the energies of the particles of interest to lie inside the passband as they enter the analyzer).

To simplify matters, we use a finite number of such voltage combinations, called optics 'states'. Table 3 lists the beam collection areas $A$, geometric factors $H$ and acceptance angles for the 'states' suitable for the electron drift measurements. For each state there is a look-up table from which the voltages that vary as a function of the polar angle are obtained. State 6 has the largest sensitivity $A$ for beams, but also a large $H$-factor for ambient electrons, and thus not a good SNR. State 3 has the highest SNR, but less than half the area $A$ of State 6. States 2 and 7 have only modest SNR, but large acceptance angles, and thus are suited for cases where there is uncertainty in $\delta$, the angle change of the returning beam. State 4 , finally, is a 'shut-down' state, to be used when fluxes are very high.

\subsubsection{Sensor}

The detector optics images electrons arriving from within the selected polar angle acceptance cone onto an annular microchannel plate (MCP) stack that is the input to the sensor. Except when the direction of the returning beam is close to the symmetry axis, only a limited azimuthal segment of the image annulus is illuminated by beam electrons, whereas the entire annulus collects ambient electrons that are incident within the optics acceptance cone and energy passband. Under command of the controller, the sensor responds only to events within a selected azimuthal range, thus complementing the polar angle selection that is performed by the optics.

The sensor collects the amplified electron pulses, which are produced by the MCP stack operated in pulse-counting mode, on an annular array of 32 discrete anodes. The sensor control structure is designed so that any four contiguous anodes in the annular array $\left(22.5^{\circ}\right.$ of azimuth) may be selected as the instantaneous field of view. For electric field measurements two adjacent anodes closest to the expected azimuthal angle of the return beam are used. Event pulses from charge pre-amplifiers of the selected two anodes are merged into a single event pulse stream in the gun-detector electronics and forwarded to the correlator.

The electrical potential of the sensor input MCP is established by the detector optical requirements. On Cluster it was possible to set this potential through a separate control voltage which allowed the detector to be used for measuring ions. Absent a requirement for measuring ions and supported by modeling of the optics, it was decided that a separate control of the MCP input voltage was not needed. The sensor interfaces with the Gun/Detector 
electronics (GDE) which forwards commands received from the EDI Controller and returns event pulses and digital status to the GDE. Analog sensor status is sampled in the GDE.

\subsection{Correlators}

To detect the beam electrons in the presence of background counts from ambient electrons and to measure their flight time, the electron beam is intensity-modulated with a binary pseudo-noise code (PNC) sequence. The modulation is achieved by changing the Wehnelt control voltage in the gun such that the beam is successively turned on and off. In order to reject detector signals from the gun in the same unit, the codes for the two gun-detector pairs are inverted relative to each other. The modulation frequency can be chosen between $65 \mathrm{kHz}$ and $4 \mathrm{MHz}$ in order to cover the range of expected time delays and to obtain adequate delay-time accuracy.

The stream of electron event pulses received by the detectors are fed in parallel into an array of counters, each one gated with its individual copy of the PNC, shifted by one chip from counter to counter, and delayed as a whole (by a variable amount) against the PNC used to modulate the outgoing beam. Event pulses that arrive while the current code element ('chip') of the PNC for a particular counter channel in the array is high, cause the counter to increment, while a low state of the current code chip will decrement the counter. Background electrons will therefore, on average, not contribute to the counts result in any of the channels of the counter array due to their random arrival times. The counter channel that is gated with the PNC matching the time of flight will increment with each arriving event pulse that was generated from a return beam electron ("signal"). In channels with non-matching delays, signal pulses will on average not contribute to the counts result. A drift and tracking control loop ('auto-track') varies the delay of the correlator codes relative to the gun code such that the signal is kept in one dedicated counter while the flight time changes as a result of changing magnetic and electric fields.

Since the time-of-flight is not known initially, the best estimate for the delay is the gyro period derived from the real-time onboard magnetic field data. Since the true time-of-flight will differ from the gyro period, the number of counters in the array must be large enough to ensure that the signal will be seen in one of the channels. On Cluster this was solved by using a 15 chip PNC together with an array of 15 counters. This ensured that the correlation peak from the return beam was guaranteed to be visible in one of the channels. The downside of this short code was that signals from electrons that gyrated more than once ('multi-runners') were also guaranteed to be picked up by the correlator if they hit the detector. A second, longer code with 127 chips was used in higher magnetic fields for discrimination of multirunners.

Due to the higher measurement requirements on MMS, higher code clock frequencies need to be used in low magnetic fields than on Cluster. In order to cover similar differences between the gyro period and actual times-of-flight at higher code clock frequencies, the counter array has been increased to 63 channels and longer codes with 255, 511 and 1023 chips have been implemented in order to reduce problems with multi-runners.

The correlator electronics resides in a RAM-based Field-Programmable Gate Array (FPGA) of the XILINX type (Virtex II XQVR600). Configuration of the FPGA is part of the start-up procedure of the instrument. As the configuration file is held in CRAM (non-volatile memory), other correlator schemes could be uploaded in flight if necessary.

\subsection{Controller}

The GDUs are controlled by flight software executing on a dedicated board inside the FIELDS CEB, the EDI Controller. Figure 6 is a sketch of the configuration of this con- 
troller. We use a SPARC V8 compliant fault tolerant LEON3 processor embedded within an ACTEL RTAX2000S FPGA, the same that is found on the Central Data Processing Unit (CDPU) boards in the FIELDS CEB. The associated memory has about $6 \mathrm{kB}$ of ROM (hard programmed into the FPGA), 2 MB of fully writable and non-volatile CRAM manufactured by BAE Systems, and 2 MB of static RAM (SRAM). Both the CRAM and SRAM are errordetected-and-corrected (EDAC). The ROM contains software for system initialization, basic housekeeping, and basic telemetry routines that allows the system to self-diagnose its status and be fully reprogrammed by command without the use of any other memory. The CRAM, which is reprogrammable, contains all the science operational software, full housekeeping routines, and the gun and optics voltage calibration tables. Some area is reserved for correlator FPGA configuration data. The static RAM is available for local storage of various parameters and variables, and also serves as telemetry-data buffer and memory for storage of burst mode or diagnostic data.

As a FIELDS subsystem, the EDI Controller resides hierarchically below the FIELDS CDPU, which acts as a relay for EDI commands and telemetry. Magnetic field information at a rate of 128 vectors/s from either the analog (AFG) or digital (DFG) flux-gate magnetometer is also received via the CDPU.

The EDI flight software for nominal operations makes use of the RTEMS (Real-Time Executive for Multiprocessor Systems) real-time operating system. Owing to the synchronous operation of the FIELDS instruments, the EDI science software can operate within a single interrupt service routine, called the 'Basis Cycle Interrupt' (BCI) routine. All other relevant timing information such as telemetry sampling boundaries and magnetometer data updates is derived from FIELDS internal timing counters, rather than separate interrupts. Together with the efficiency of the LEON3 RISC architecture compared to the Sandia 3300 processor that was used on Cluster, this allowed to reduce the Basis Cycle from $4 \mathrm{~ms}$ (on Cluster) to $2 \mathrm{~ms}$, leading to shorter response times in the detection of the return beam in electric field mode. Every BCI, the Controller uses the information it receives from the magnetometer and the GDUs to calculate new parameters for directing the beams and the detectors, and to establish and maintain beam coding and tracking patterns. Tracking tasks that operate on longer time scales, such as slowly changing the beam current or modifying the detector's basic optics state, are implemented as RTEMS tasks and are executed on a less frequent basis.

In electric field mode, the EDI processor transfers the programmed gun firing directions (for beams returning to the detectors), plus the timing information from the correlators, to the necessary telemetry data so that the resultant drift-step direction and magnitude can be determined on the ground. In ambient mode, electron counts accumulated over time intervals depending on the respective telemetry product are transmitted together with pitch angle flags and detector look direction.

\subsection{Resources}

The GDU mass is $5.96 \mathrm{~kg}$. The orbit average GDU power consumption is $5.0 \mathrm{~W}$ in Electric Field Mode and $3.5 \mathrm{~W}$ in Ambient Mode. The science data rates allocated to EDI are 1800 bits/s for Fast Survey and 71936 bits/s for Burst telemetry. 


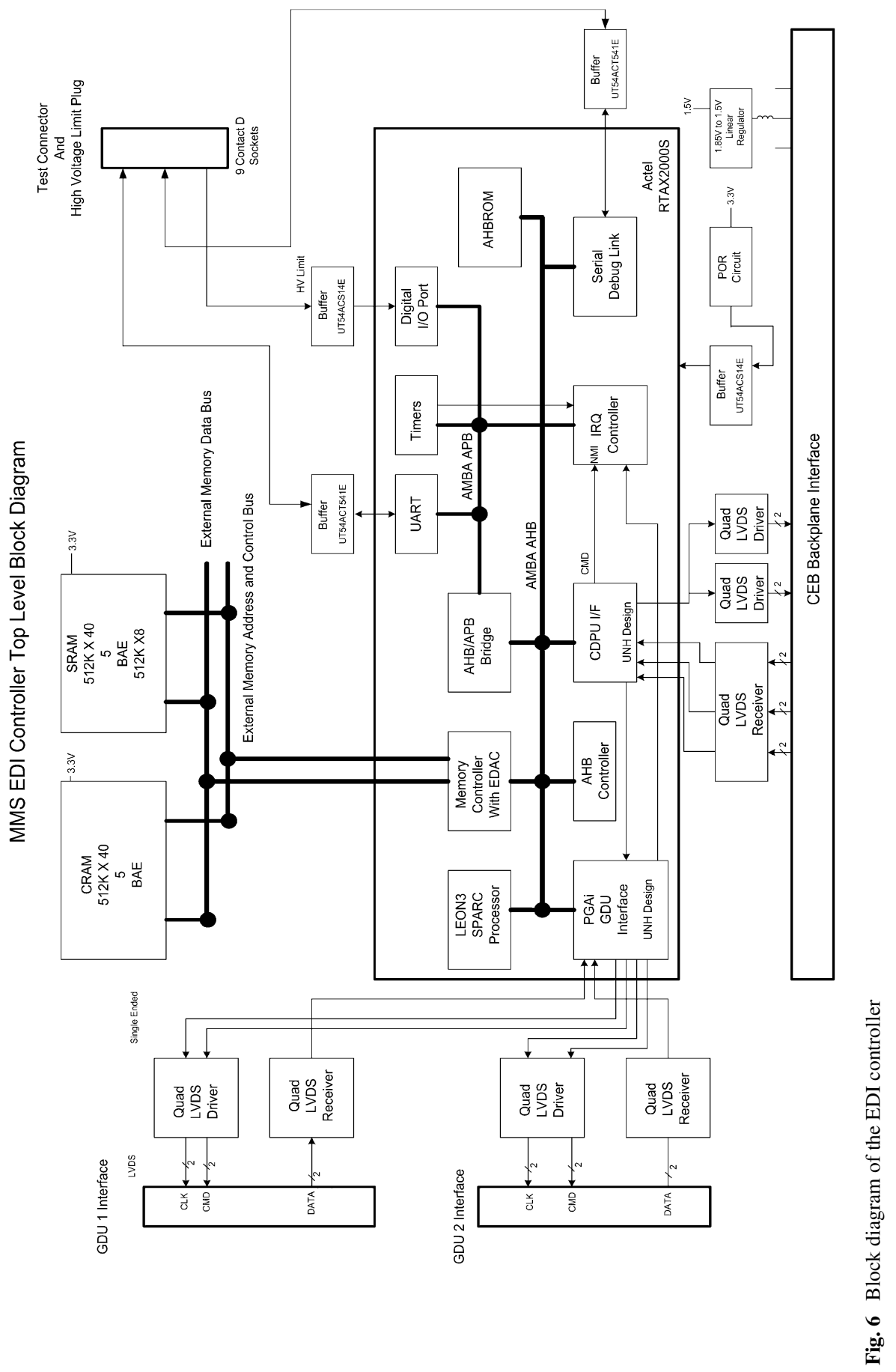




\section{Calibrations and Simulations}

\subsection{Gun Calibrations}

The electron guns were calibrated at IWF in a dedicated facility at electron energies of 0.5 and $1.0 \mathrm{keV}$ (and $250 \mathrm{eV}$ for some guns), up to the maximum deflection angle of 96 degrees and over the entire 360 degrees in azimuth, with a grid size of 2 degrees below a polar angle of 70 degrees, and a grid size of 1 degree for higher polar angles. The pointing accuracy is approximately 0.5 degrees. Information on beam profiles and beam intensity control were also gathered during this process. The facility and operational mode was nearly identical to that employed for Cluster and as described in Paschmann et al. (1998).

\subsection{Optics Simulations and Verification}

The large number of potential surfaces of the EDI optics provides a large parameter space for operation of the instrument. In order to develop operation modes for various plasma parameters that occur over different parts of the orbit of MMS, extensive simulations of the EDI optics have been performed to determine the appropriate sets of voltages to achieve the desired response to the incoming electron beam. Optimization of optics states is driven by finding the best signal-to-noise ratio for the returning beam, allowing for good correlator response and rapid identification that the return beam has been found. In this context the signal-to-noise ratio is determined by the count level of the return beam relative to the background signal from ambient electrons in the plasma external to the spacecraft. The electron beam signal is determined by the total area of the optics response to any returning electron which intercepts this area. The noise, or background signal, is determined by the overall geometric factor of the optics. Consequently, optimization results in maximizing the total area to geometric factor ratio of the optics.

The baseline optics state for MMS operations is one that we carry over from the Cluster mission and is known as "Optics State 7". This state provides a good area to geometric factor ratio which proved to work well in most plasma regimes. From the baseline Cluster optics state, we have further optimized the voltages to account for the changes in design of the EDI from Cluster to MMS as well as the much-improved state of computation which allows for more accurate simulation of the exact optics design.

To perform these simulations, we have utilized the commercial Lorentz software suite which provides both the electric field solution as well as the electron trajectory integration through the electric field solution and structure of the optics. For any given optics state look direction, several thousand simulated trajectories are calculated and the position of those which would hit the microchannel plates are recorded. The input trajectories are varied over input angle and location so that all possible successful trajectories over the full range of angles and source locations are bounded by the simulation. With the simulation results, we can then build a map of input angle vs. pad number (over the 32 pads upon which counts are detected) for a given look direction of the optics state.

The simulations are complemented by characterizations of the detectors that were performed on all flight units during GDU testing. The electron source for these characterizations was a broad beam that was generated using a UV backlit thin plate with chrome coating. The plate was biased negatively at the desired voltage (energy) and photoelectrons emanating from the plate were accelerated towards a grid at ground potential between the plate and the GDU. The GDU was mounted on a manipulation table that allowed the rotation of the GDU 360 degrees around its symmetry axis and pitching the GDU towards/away from the 
beam so that incident beam angles of 0 to 110 degrees relative to the symmetry axis could be achieved. The characterizations performed consisted of roll tests around the symmetry axis for selected combinations of optics look-angle voltages and associated pitch angles. These tests demonstrated the desired degree of uniformity of the integrated detector system and established the dependency of the effective beam area on polar angle for monodirectional and monoenergetic beams. Another set of tests was run to demonstrate and characterize the polar acceptance in optics states 7 and 4 . In these tests the GDU was pitched, for a number of look directions, from 0 to 110 degrees at fixed roll angle and fixed optics look direction. In addition to these tests, adequate rejection of UV light was demonstrated. All tests were performed at $500 \mathrm{eV}$ and $1 \mathrm{keV}$ beam energy and included variations of the beam energy by $10 \%$ relative to the energy setting of the detector.

Figure 7 shows a comparison between the simulated response and measured response for the three look directions, from left to right, of $\theta=30^{\circ}, 60^{\circ}$, and $90^{\circ}$ which illustrate the type of comparison we perform. In general, the agreement is quite good in terms of shape and extent of the response for these three angles. There is a small offset of $\sim 3^{\circ}-5^{\circ}$ between the simulated response and that of the measured response which we believe to be due to alignment error in the system for measuring the response in the laboratory. Note that as the look direction decreases, a set of 'wings' begins to appear at lower angles. This effect is due to the fact that at smaller angles, the absolute angular separation between the direction defined by the center of the pad and the outward direction at that polar angle and the pad next to it decreases, spreading the beam across adjacent pads. A key point to note is that the response of the optics appears narrow as a function of pad number and relatively broad as a function of polar angle, each pad is $11.25^{\circ}$ wide in the azimuthal direction so that the response in the azimuthal direction is actually comparable to that in the polar direction.

This set of comparisons between measurement and simulation provides verification that the simulation is accurate and can be used with confidence for developing other optics states that are optimized for other plasma parameters. The simulations have also been used to develop the most useful ambient mode that provides good geometric factor and somewhat narrower angular response appropriate for using the EDI as a detector of the background plasma. Verification of the simulation also enables updates of the EDI optics settings based upon on-orbit experience, giving us the ability to further optimize the electron beam detection system for the best results.

\subsection{Tracking Simulator}

The electric field mode of EDI is implemented as a closed loop operation that controls the gun, optics, sensor and correlator of both GDUs based on real time magnetic field data from one of the flux-gate magnetometers, recent correlator data that allow the recognition of return beams and determination of associated times-of-flight, as well as longer term averaged statistical data about background count rates and return beam tracking success. Due to the large scale of the electron gyroradius, the in-orbit environment cannot be replicated in ground testing. For the purpose of validating the control algorithms in the EDI flight software, a tracking simulator was developed. This simulator consists of GDU emulation hardware that interfaces with the Controller in the same fashion as real GDUs do, and of associated software that calculates background and return beam signal count rates based on the instantaneous gun, optics and sensor settings controlled by the EDI flight software. The calculations in the simulator make use of real gun calibration and optics characteristics data, and of reference tables that contain proper beam firing and detector look directions as well as corresponding times-of-flight for chosen magnetic and electric field time series. 

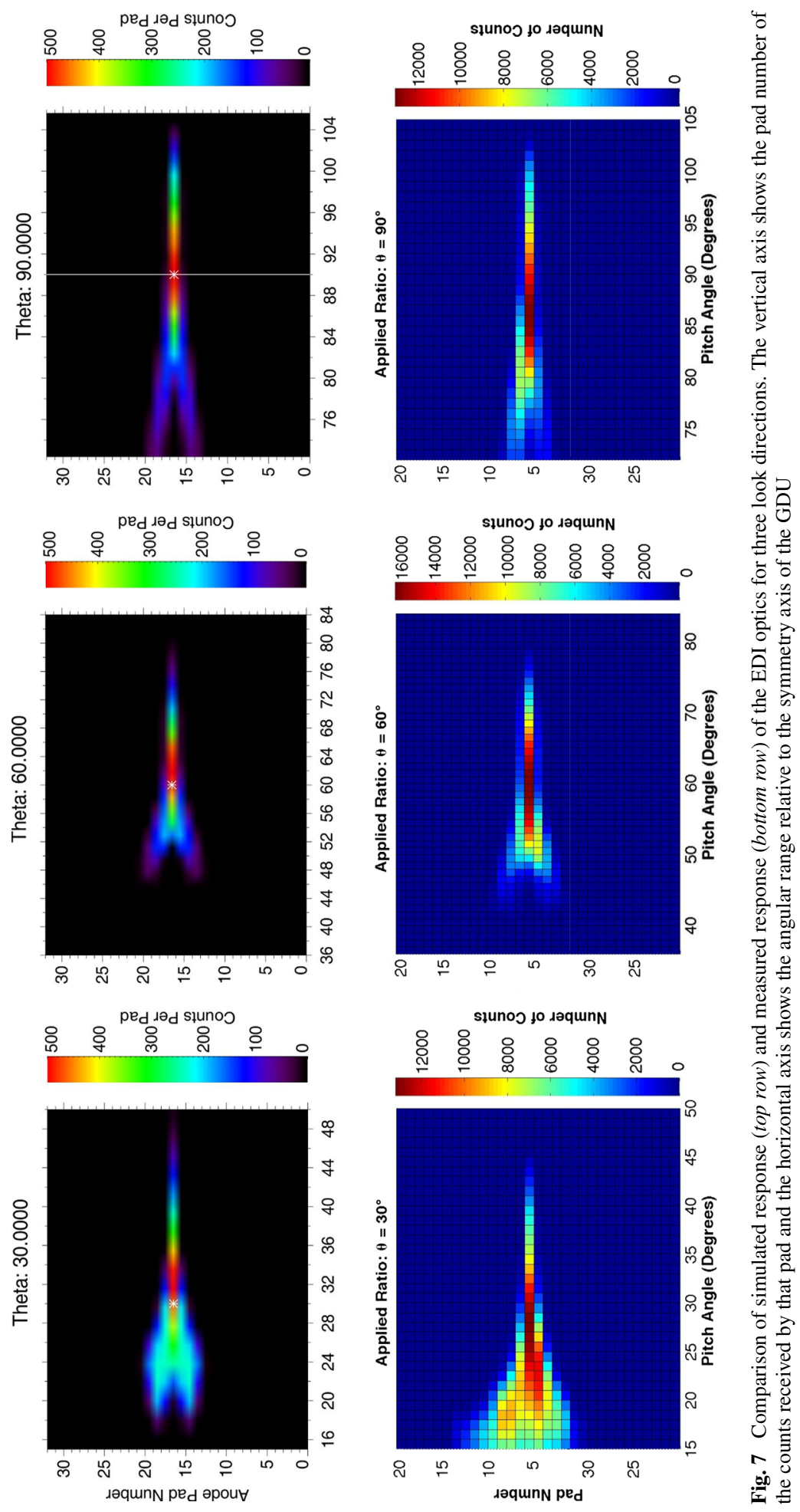
These time series as well as fluxes of ambient electrons are taken from actual data measured with instruments on previous missions. The simulator also implements the flux-gate magnetometer interface to the FIELD CDPU through which magnetic field data get updated during a particular simulation run in the same fashion as in the real flight hardware in the FIELDS CEB.

The calculated signal and background count rates can be fed back to the EDI flight software in one of two modes. Either the simulator uses an assumed average performance of the correlator and sends back simulated correlator data such as correlated counts and measured times-of-flight. Or, more realistically, the simulator directs hardware event pulsers to generate real streams of signal and background pulses. The signal event pulse stream is modulated with a pseudo-noise code as it would be used in a real gun, but properly delayed by the timeof-flight in order to simulate the phase delay of the code imposed on a real return beam. The correlators in the GDU emulators generate data that are sent back to the EDI Controller and are analyzed by the flight software to determine the commands for the gun, optics, sensor and correlator in the next time step.

Several reference tables exist to simulate different environments such as magnetosphere, magnetosheath and magnetopause crossing cases. Running through these cases we can optimize the flight software and its underlying algorithms for their ability to cope with realistic magnetic and electric fields and their variations.

\section{Science Modes and Data Products}

\subsection{Electron Drift Measurements}

Our basic tracking mode, referred to as the nominal Electric Field mode, also called Windshield-Wiper (WW) Mode on Cluster, uses an acquisition mode, sweeping the beams of the each GDU independently in the plane perpendicular to $\mathbf{B}$, until each beam has hit its target, $S 1$ and $S 2$. Once this has occurred, we will track $S 1$ and $S 2$ independently, by sweeping the beams rapidly back and forth across them, in the plane perpendicular to $\mathbf{B}$, so that the beam hits (or is very near) $S$ all the time. This is a substantial improvement over earlier modes on Cluster, called GEOS and Rapid Spin (RS) schemes (see Paschmann et al. 1998), in which the beam is fired at $S$ only when either the spacecraft rotation or the passive angular sweep is at the correct phase.

To recognize that the beam is returning to the detector, the flight software (FSW) constantly (once every $2 \mathrm{~ms}$ ) calculates the signal-to-noise ratio from quantities pre-computed and returned by the correlator hardware. If that ratio exceeds some (selectable) limit, it is assumed that the beam is striking the detector. Initially the FSW steers each detector to look at a direction anti-parallel to the emission direction of the associated beam. This works as long as the aberration angle $\delta$ introduced above is less than the acceptance angle of the detector. For larger aberration angles the detector look-direction must be offset appropriately. This complicates target acquisition.

The 'windshield-wiper' software, which keeps the target direction tracked in angle space, is interwoven intricately with the time-tracking software, which keeps the time-of-flight information available. As noted earlier, the beams are modulated with a coded waveform. Correlating the received signal, after a delay corresponding to the time of flight, with the original coded signal, has two important functions. First, correlating the return signal with the fired signal allows a significant increase in signal-to-noise ratio. Second, the delay of 
the channel holding the largest number of counts, if above the signal-to-noise ratio limit, corresponds to the time of flight of the electron beam.

In the windshield-wiper mode telemetered data consist of the firing angles when the two beams were on target, and the two associated electron times-of-flight, plus timing and quality information. With the allocated telemetry rate it is possible to transmit this set of measurements 16 times per second in Fast Survey, and over 100 times per second in BURST telemetry (see Torbert et al. 2014 for definitions of these modes on MMS).

\subsection{Ground Processing (Bestarg)}

The Bestarg software analyzes a set of beam data consisting fundamentally of GDU positions, firing angles, and times-of-flight (ToF) from the windshield wiper mode described above. With this information, Bestarg uses the triangulation and time-of-flight methods (Sects. 3.1 and 3.2) to search for the target (Fig. 3). Both methods are undertaken in the gun firing plane - the plane perpendicular to B (BPP) — and result in an estimate of the drift step. Finally, the drift step is passed on to calculate the electric field (Eqs. (2) and (1) above).

During an analysis interval (typically $5 \mathrm{~s}$ ), a single BPP is created from an averaged magnetic field. Instantaneous beam information is used directly in this BPP to determine the drift step via the triangulation method. Candidate beam intersections (drift steps) are modeled by a grid of drift steps. Chi-squared statistics are performed at each grid point using the difference between GDU and model firing angles and the intrinsic beam uncertainty. At first, outliers are included, given that the distribution of model firing angles has a broad tail. Later, outliers are discarded, and the distribution is assumed to be normal. A finer grid is used and chi-squared statistics hone in on the true drift step. Thus, the fine grid forms a probability surface from which to determine the drift step and its associated error.

Results from the triangulation method are supplemented by considering "multirunners" and by the time-of-flight method. Multirunners are electrons that return to the spacecraft after more than one gyration and have drifted by a distance that is a multiple of the drift step. The same model drift steps have an associated time-of-flight that can be compared with that of each beam. A similar chi-squared analysis results in a most probable drift step.

Once found, the drift step can be used with Eq. (1) to determine the electric field. This electric field does not depend on local spacecraft effects and is used in later FIELDS software (see Torbert et al. 2014, this issue) to calibrate the double probe instruments.

\subsection{Ambient Particle Measurements}

The EDI detectors are capable of measuring electron fluxes at very high time resolution. Each of the two detectors can be commanded to look in any direction over greater than a $2 \pi$ steradian hemisphere, and since the two detectors are mounted on opposite sides of the spacecraft with their symmetry axes pointing in opposite directions, full-sky surveys can be achieved without relying on spacecraft spin to complete the coverage of phase space. In addition, since the $\mathbf{B}$ field is known to the EDI controller via the on-board magnetometer data, specialized surveys can be performed in coordinates fixed with $\mathbf{B}$ and they can be performed continuously.

\subsection{Magnetometer Offsets}

EDI beam data, namely electron times-of-flight (TOFs) and beam firing directions (BD), will be used for spin axis offset determination of the flux-gate (FG) magnetometers AFG and 
DFG. Both quantities can be used separately to perform this calibration task (see Plaschke et al. 2014). TOFs are inversely proportional to the strength of the ambient magnetic field. Hence, differences in field strength measured by a FG magnetometer and derived from EDI TOFs can be attributed to spin axis offsets, if the FG is otherwise accurately calibrated. However, as discussed in Nakamura et al. (2014), EDI TOFs are also subject to GDU and EDI mode specific offsets that have to be corrected first. For this task, FG and EDI data may be compared when the magnetic field is close to the spacecraft spin plane, so that FG field strength observations are least affected by uncertainties in spin axis offset.

BDs have to be perpendicular to the ambient magnetic field for electrons to return to the spacecraft. Consequently, deviations of angle $\alpha$, which is the angle between FG measured magnetic field vectors and EDI BDs, from $90^{\circ}$ can be attributed to spin axis offsets. BDs are not affected by TOF offsets. However, the EDI BD and FG magnetic field vector coordinate systems are required to coincide precisely. The systems can be adjusted by minimizing $\left(\alpha-90^{\circ}\right)^{2}$ for BDs in the spin plane, as the corresponding angles $\alpha$ are least affected by spin axis offset uncertainties. A more detailed explanation of the offset corrections is contained in Torbert et al. (2014, this issue).

\section{Conclusion}

The Electron Drift Instrument on MMS is the latest manifestation of this technique on space missions. It has enhanced capacity, over Cluster, to track the drift step and thus return accurate electric field measurements over more varied plasma conditions and magnetic field variations. With its inherent geometric accuracy, EDI will provide critical calibrations to the double probes on MMS, offset corrections to the flux-gate magnetometers, and very high time resolution electron fluxes. Because of these contributions, the stringent requirements of MMS to measure precisely reconnection and parallel electric fields, and to follow rapidly varying plasma distribution functions can be met.

Acknowledgements We are indebted to the following individuals for the part they have played in instrument manufacture, testing and qualification: at UNH, K.L. Singer, J. Googins, T. Jones, S. Ellis, J. Levasseur, A. Bolton, P. Demaine, M.J. Golightly, K. Frederick-Frost, J. Nolin. At the University of Iowa, S. Kutcher, M. Fountain, L. Detweiler, J. Howard, M. Miller, and at IWF, S. Neukirchner and H. Hartl. This work was supported by the National Aeronautics and Space Administration under contract 499878Q.

Open Access This article is distributed under the terms of the Creative Commons Attribution 4.0 International License (http://creativecommons.org/licenses/by/4.0/), which permits unrestricted use, distribution, and reproduction in any medium, provided you give appropriate credit to the original author(s) and the source, provide a link to the Creative Commons license, and indicate if changes were made.

\section{References}

J.L. Burch, T.E. Moore, R.B. Torbert, B.L. Giles, Magnetospheric multiscale overview and science objectives. Space Sci. Rev. (2014, this issue). doi:10.1007/s11214-015-0164-9

J. Egedal, W. Daughton, A. Le, Large-scale electron acceleration by parallelelectrical fields during magnetic reconnection. Nat. Phys. (2012). doi:10.1038/NPHYS2249

F. Melzner, G. Metzner, D. Antrack, The GEOS electron beam experiment. Space Sci. Rev. 4, 45 (1978)

R. Nakamura, F. Plaschke, R. Teubenbacher, L. Giner, W. Baumjohann, W. Magnes, M. Steller, R.B. Torbert, H. Vaith, M. Chutter, K.-H. Fornacon, K.-H. Glassmeier, C. Carr, Interinstrument calibration using magnetic field data from the flux-gate magnetometer (FGM) and electron drift instrument (EDI) onboard Cluster. Geosci. Instrum. Method. Data Syst. 3, 1-11 (2014). doi:10.5194/gi-3-1-2014 
G. Paschmann, F. Melzner, R. Frenzel, H. Vaith, P. Parriger, U. Pagel, O.H. Bauer, G. Haerendel, W. Baumjohann, N. Sckopke, R.B. Torbert, B. Briggs, J. Chan, K. Lynch, K. Morey, J.M. Quinn, D. Simpson, C. Young, C.E. McIlwain, W. Fillius, S.S. Kerr, R. Maheu, E.C. Whipple, The electron drift instrument for cluster. Space Sci. Rev. 79, 233 (1998)

G. Paschmann, C.E. McIlwain, J.M. Quinn, R.B. Torbert, E.C. Whipple, The electron drift technique for measuring electric and magnetic fields, in Measurement Techniques in Space Plasmas: Fields. Geophysical Monograph, vol. 103 (AGU, Washington, 1998)

F. Plaschke, R. Nakamura, H.K. Leinweber, M. Chutter, H. Vaith, W. Baumjohann, M. Steller, W. Magnes, Flux-gate magnetometer spin axis offset calibration using the election drift instrument. Meas. Sci. Technol. (2014, submitted)

P.L. Pritchett, F.S. Mozer, Asymmetric magnetic reconnection in the presence of a guide field. J. Geophys. Res. 114, JA014343 (2009)

J.M. Quinn, C.E. McIlwain, G. Haerendel, F. Melzner, D. Cauffman, Measurement of vector electric fields using electron test particles. Eos Trans. AGU 60, 918 (1979)

W. Riedler, K. Torkar, F. R“udenauer et al., Active spacecraft potential control. Space Sci. Rev. 79, 271 (1997).

R.B. Torbert et al., The FIELDS instrument suite on MMS: scientific objectives,measurements, and data products. Space Sci. Rev. (2014). doi:10.1007/s11214-014-0109-8

K. Torkar, R. Nakamura, M. Tajmar, C. Scharlemann, H. Jeszenszky, G. Laky, G. Fremuth, C.P. Escoubet, K. Svenes, Active spacecraft potential control investigation. Space Sci. Rev. (2014, this issue). doi:10.1007/s11214-014-0049-3 